\title{
Microstructural integrity of the cingulum is related to verbal memory performance in elderly with cerebral small vessel disease The RUN DMC study
}

\author{
H.M. van der Holst ${ }^{\text {a,b, } 1}$, A.M. Tuladhar ${ }^{\text {a,b, } 1}$, A.G.W. van Norden ${ }^{\text {a,b }}$, K.F. de Laat ${ }^{\text {a,b }}$, I.W.M. van Uden ${ }^{\text {a,b }}$, \\ L.J.B. van Oudheusden ${ }^{\text {a,b }}$, M.P. Zwiers ${ }^{\text {b,c }}$, D.G. Norris ${ }^{\text {b }}$, R.P.C. Kessels ${ }^{\text {b, d,e }}$, F.E. de Leeuw ${ }^{\text {a, b,* }}$ \\ a Department of Neurology, Donders Institute for Brain, Cognition and Behaviour, Centre for Neuroscience, Radboud University Nijmegen Medical Centre, 6500 HB Nijmegen, \\ The Netherlands \\ ${ }^{\mathrm{b}}$ Centre for Neuroimaging, Donders Institute for Brain, Cognition and Behaviour, Radboud University Nijmegen, 6500 HE, The Netherlands \\ ${ }^{c}$ Department of Psychiatry, Donders Institute for Brain, Cognition and Behaviour, Centre for Neuroscience, Radboud University Nijmegen Medical Centre, 6500 HB Nijmegen, \\ The Netherlands \\ ${ }^{\mathrm{d}}$ Department of Medical Psychology, Donders Institute for Brain, Cognition and Behaviour, Radboud University Nijmegen Medical Centre, 6500 HB Nijmegen, The Netherlands \\ e Department of Geriatrics, Donders Institute for Brain, Cognition and Behaviour, Radboud University Nijmegen Medical Centre, 6500 HB Nijmegen, The Netherlands
}

\section{A R T I C L E I N F O}

\section{Article history:}

Accepted 24 September 2012

Available online 29 September 2012

\section{Keywords:}

Cingulum

Verbal memory

Diffusion tensor imaging

Hippocampus

Cerebral small vessel disease

\begin{abstract}
A B S T R A C T
Background: Cerebral small vessel disease (SVD) is related to verbal memory failures. It is suggested that early white matter damage, is located, among others, in the (posterior) cingulum at an early stage in neurodegeneration. Changes in the microstructural integrity of the cingulum assessed with diffusion tensor imaging (DTI), beyond detection with conventional MRI, may precede macrostructural changes and be related to verbal memory failures. Objective: To investigate the relation between cingular microstructural integrity and verbal memory performance in 503 non-demented elderly with cerebral SVD.

Methods: The RUN DMC study is a prospective cohort study in elderly (50-85 years) with cerebral SVD. All participants underwent T1 MPRAGE, FLAIR and DTI scanning and the Rey Auditory Verbal Learning Test. Mean diffusivity (MD) and fractional anisotropy (FA) were assessed in six different cingular regions of interests (ROIs). Linear regression analysis was used to assess the relation between verbal memory performance and cingular DTI parameters, with appropriate adjustments. Furthermore a TBSS analysis of the whole brain was performed to investigate the specificity of our findings.

Results: Both our ROI-based and TBSS analysis showed that FA was positively related to immediate memory, delayed recall, delayed recognition and overall verbal memory performance of the cingulum, independent of confounders. A similar distribution was seen for the inverse association with MD and verbal memory performance with TBSS analysis. No significant relations were found with psychomotor speed, visuospatial memory and MMSE. When stratified on hippocampal integrity, the MD and FA values of the cingular ROIs differed significantly between participants with a good and poor hippocampal integrity.

Conclusion: Microstructural integrity of the cingulum, assessed by DTI, is specifically related to verbal memory performance, in elderly with SVD. Furthermore we found that when the integrity of the hippocampus is disrupted, the cingulum integrity is impaired as well.
\end{abstract}

(c) 2012 Elsevier Inc. All rights reserved.

\section{Introduction}

Cerebral small vessel disease (SVD) includes white matter lesions (WML) and lacunar infarcts and is a frequent finding on magnetic resonance imaging (MRI) scans of elderly people (de Leeuw et al., 2001). Several patient- and population based studies have shown that

\footnotetext{
* Corresponding author at: Department of Neurology, Donders Institute for Brain, Cognition and Behaviour, Centre for Neuroscience, Radboud University Nijmegen Medical Centre, 6500 HB Nijmegen, The Netherlands. Fax: + 31243541122. E-mail address: H.deLeeuw@neuro.umcn.nl (F.E. de Leeuw).

1 Both authors contributed equally to this work.
}

cerebral SVD is related to verbal memory failure and may eventually result in cognitive decline and dementia in some (de Groot et al., 2000, 2002; Vermeer et al., 2003). This is thought to be the result of disruption of white matter (WM) tracts. WM damage in Alzheimer dementia ( $A D$ ) has been identified both in postmortem studies (Brun and Englund, 1986; Gouw et al., 2008) as well as in vivo MRI studies (Acosta-Cabronero et al., 2010; Gouw et al., 2008). A more detailed investigation of the white matter using neuroimaging can be revealed by diffusion tensor imaging (DTI), a noninvasive MRI technique, which provides detailed information on the microstructure and integrity of white matter fiber tracts (Basser et al., 1994; Pierpaoli et al., 1996). Two DTI parameters are of special interest: 
mean diffusivity (MD), a measure of water diffusion averaged in all spatial directions, and fractional anisotropy (FA), which provides information about the directionality of water diffusion. Loss of microstructural integrity is typically accompanied by a decrease in FA and/ or an increase in MD (Charlton et al., 2006). There is increasing evidence that DTI parameters are an earlier marker of cognitive decline in comparison to volume measures (Kantarci et al., 2005; Müller et al., 2007). The cingulum bundle, a white matter bundle which connects the medial temporal lobe (MTL) structures (e.g. hippocampus) and the posterior cingulate cortex, is an important structure for memory function, especially verbal memory performance (Fellgiebel et al., 2005; Sepulcre et al., 2008; Sexton et al., 2010). Many DTI studies in mild cognitive impairment $(\mathrm{MCI})$ and $\mathrm{AD}$ patients have shown that early WM damage, is located, among others, in the (posterior) cingulum at an early stage in neurodegeneration and may be a key marker of early pathology (Catheline et al., 2010; Choo et al., 2010; Chua et al., 2009; Fellgiebel et al., 2005; Kiuchi et al., 2009; Nakata et al., 2008; Xie et al., 2005; Zhang et al., 2007). To our best knowledge all studies done so far had relatively small sample sizes $(n<249)$, used mostly only $\mathrm{MCI}$ and $\mathrm{AD}$ patients, and did not or only limited adjust for possible confounders. Furthermore most of these studies used either a region of interest (ROI) approach or a voxel-based morphometry (VMB) analysis, which have both methodological limitations. Tract-based spatial statistics (TBSS) is a relatively new method, using only those white matter voxels that are in the skeleton (core) of the brains connectional architecture, which enables a robust voxelwise analysis of the microstructural integrity of white matter and this can be accurately matched across subjects (Smith et al., 2006).

In this study we combined a ROI-based and TBSS approach in order to investigate the relation between cingulum integrity and verbal memory performance in non-demented elderly with cerebral SVD. We hypothesized that loss of microstructural integrity of the cingulum is related to impaired verbal memory performance. We examined this in six different ROIs from the posterior to the anterior cingulum in order to get information about regional distribution patterns in respect to verbal memory and performed a TBSS analysis of the whole brain as well, to investigate the specificity of our findings. In addition we investigated the role of hippocampal integrity on cingulum integrity to identify if cingular structural integrity is related to hippocampal pathology. This study is part of the Radboud University Nijmegen Diffusion tensor and Magnetic resonance imaging Cohort (RUN DMC) study that included 503 non-demented, independently living elderly with cerebral SVD, aged between 50 and 85 years.

\section{Methods}

\section{Study population}

The Radboud University Nijmegen Diffusion tensor and Magnetic resonance imaging Cohort (RUN DMC) study prospectively investigates the risk factors and clinical consequences of brain changes among 503 non-demented elderly, aged between 50 and 85 years, with cerebral SVD. The selection procedure of the participants and study protocol were described in detail previously (van Norden et al., 2011a).

In short, on the basis of established research criteria, SVD was defined as the presence of lacunar infarcts and/or WML (Erkinjuntti, 2002). Accordingly, in 2006, consecutive patients referred to the Department of Neurology between October 2002 and November 2006, were selected for participation. Inclusion criteria were: (a) age between 50 and 85 years; (b) cerebral SVD on neuroimaging (WML and/or lacunar infarcts). The main exclusion criteria were dementia (American Psychiatric Association, 2000), (psychiatric) disease interfering with cognitive testing or follow-up, WML or SVD mimics and MRI contraindications or known claustrophobia.

From 1,004 invited individuals by letter, 727 were eligible after contact by phone of whom 525 agreed to participate. In 22 individuals exclusion criteria were found during their visit to our research center, yielding a response of $71.3 \%$ (503/705). For the present study, 63 subjects were additionally excluded because of territorial infarcts $(n=59)$ and inadequate quality of the MRI image $(n=4)$. All participants signed an informed consent form. The Medical Review Ethics Committee region Arnhem-Nijmegen approved the study.

\section{Measurement of cognitive function}

Cognitive function was assessed by a standardized neuropsychological test battery performed by two trained investigators (AvN and KdL) and has been described in detail elsewhere (van Norden et al., 2011a). For this study, the Mini-Mental State Examination (MMSE) (range, 0-30) (Folstein et al., 1975) was used as an index of overall cognitive performance. The three-trial version of the Rey Auditory Verbal Learning Test (RAVLT) (van der Elst et al., 2005) was administered to examine episodic memory formation. To evaluate speed of mental processes we used the Stroop test (Houx et al., 1993; Stroop, 1935), the Paper-Pencil Memory Scanning Task (Sternberg, 1969), and the Symbol-Digit Substitution Task (Lezak, 1976). The Rey Complex Figure Test (RCFT) (Osterrieth, 1944) was included as an index of visuospatial memory.
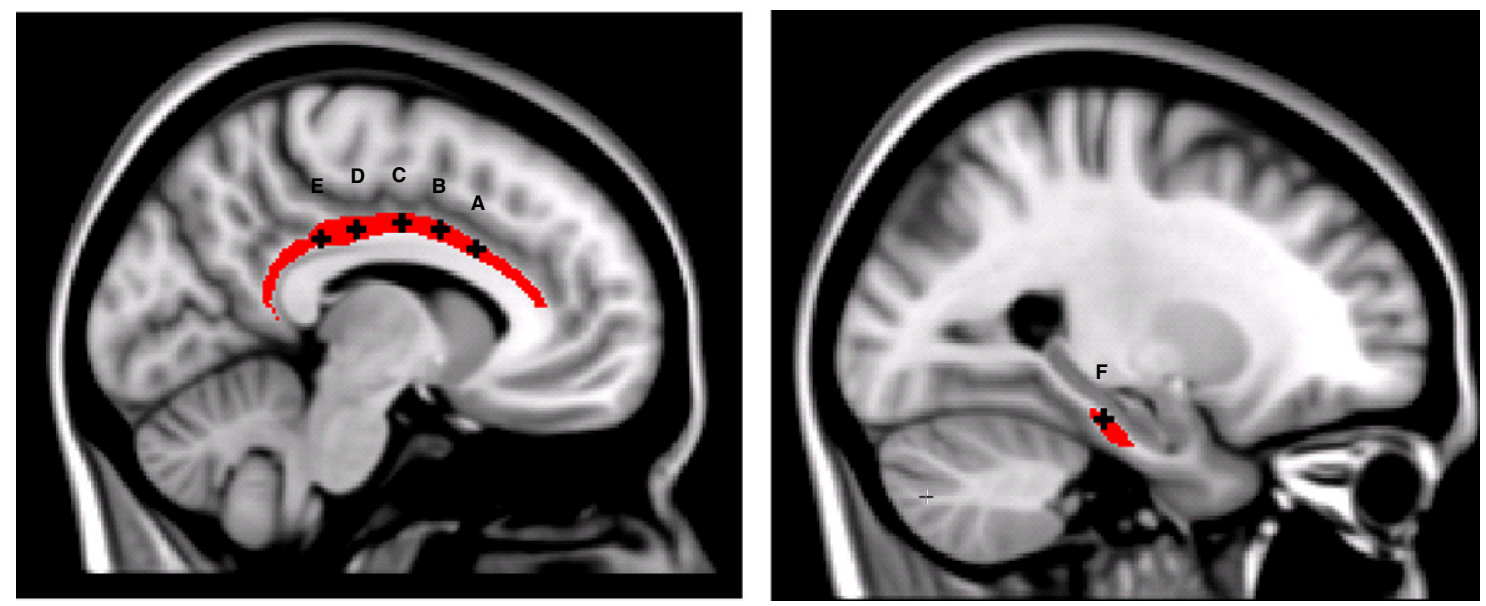

Fig. 1. Location of the six (A-F) regions of interest (ROIs) in the left cingulum in a sagittal plane. 


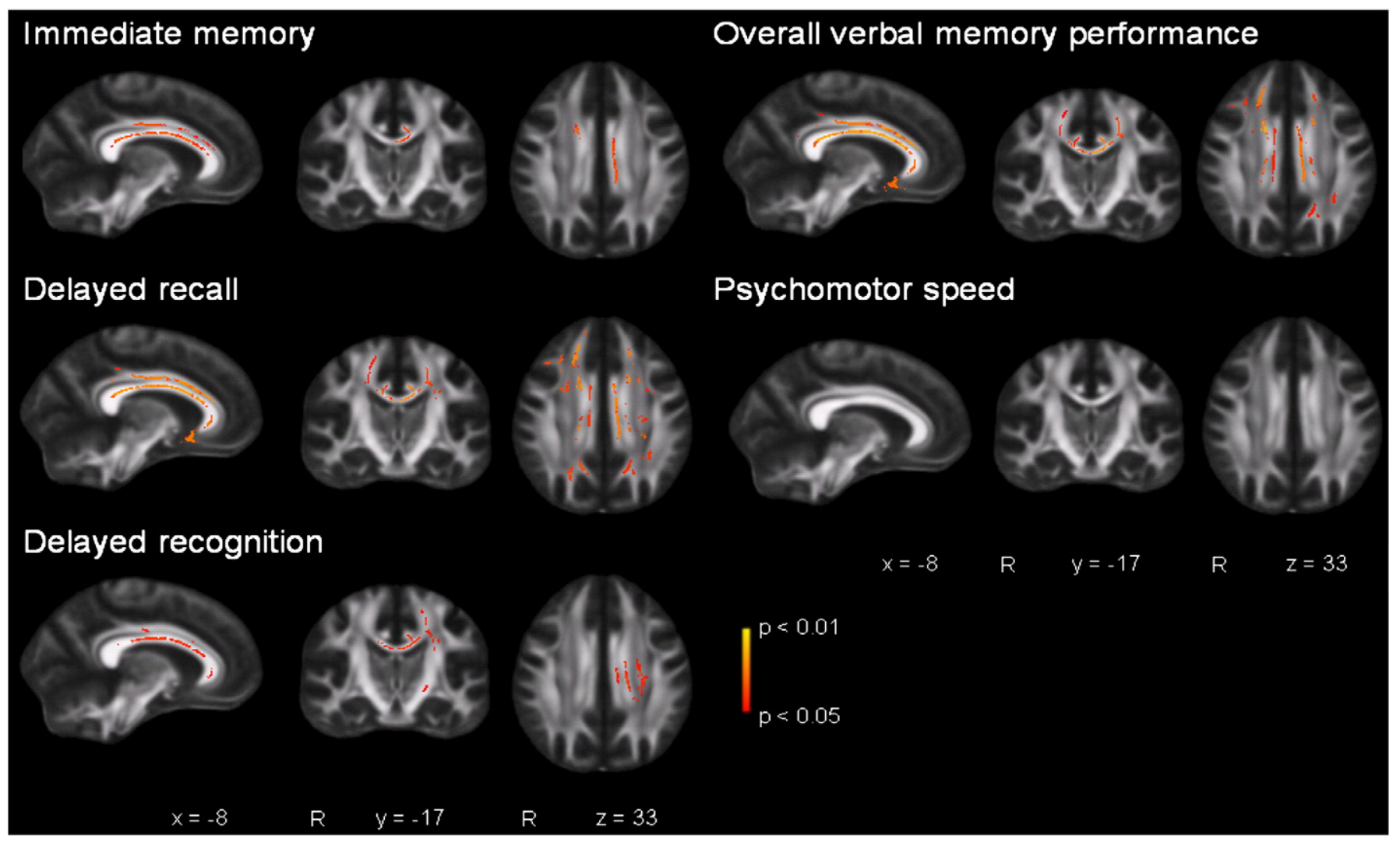

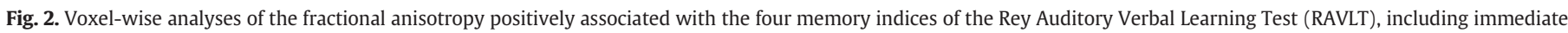

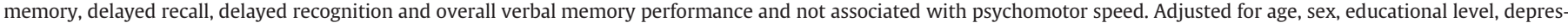
sive symptoms, normalized total brain volume, normalized hippocampal volume, WML volume and the number of lacunar infarcts.

We defined four memory indices based on RAVLT performance (overall verbal memory performance, immediate recall, delayed recall, and delayed recognition), as described previously (Vakil and Blachstein, 1993; van Norden et al., 2011b). Immediate recall was calculated by the mean of the total number of words remembered in the three learning trials of the RAVLT. Delayed recall was the number of words recalled 30 min after the learning trials. The delayed recognition score was calculated by computing the total of each correctly recognized word (the 15 target words among 15 new distracter items) 30 min after the learning trials. Performance across tests was made comparable by transforming the raw test scores into Z-scores as described elsewhere (van Norden et al., 2011b) for which the assumption of normality of the distribution was examined. For data reduction purposes, a compound score for overall verbal memory performance was calculated, as described previously (de Groot et al., 2000), by taking the mean of two Z-scores from the RAVLT: one for the added scores on three learning trials of this test and one for the delayed recall of this test.

For psychomotor speed and visuospatial memory we also calculated compound scores. Psychomotor speed was calculated as the mean of the Z-scores of the 1-letter subtask of the Paper-Pencil Memory Scanning Task, the reading subtask of the Stroop test and the Symbol-Digit Substitution Task (van Norden et al., 2011c). Visuospatial memory is a compound score of the mean of the Z-scores of the immediate recall trial and the delayed recall trial of the Rey Complex Figure Test.

\section{Conventional MRI scanning protocol}

All participants underwent a 1.5-T MRI scanning on the same Magnetom scanner (Siemens, Erlangen, Germany). The scanning protocol includes whole brain 3D T1 magnetization-prepared rapid gradient-echo (MPRAGE) sequence (TR/TE/TI 2250/3.68/850 ms; flip angle $15^{\circ}$; voxel size $\left.1.0 \times 1.0 \times 1.0 \mathrm{~mm}\right)$; a fluid-attenuated inversion recovery (FLAIR) pulse sequences (time repetition [TR]TE/TI $9000 / 84 / 2200 \mathrm{~ms}$; voxel size $1.0 \times 1.2 \times 6.0 \mathrm{~mm}$ (including slice gap of $1 \mathrm{~mm}$ )); DTI (TR/TE $10100 / 93 \mathrm{~ms}$; voxel size $2.5 \times 2.5 \times 2.5 \mathrm{~mm}$; 4 unweighted scans, 30 diffusion weighted scans, with non colinear orientation of the diffusion-weighting gradient, and b-value $900 \mathrm{~s} / \mathrm{mm} 2$ ). For more details of our MRI protocol we refer to our study rationale and protocol published in BioMed Central Neurology in 2011 (van Norden et al., 2011a).

\section{Conventional MRI analysis}

One experienced investigator, blinded to clinical data (IvU), manually segmented the left and right hippocampus on the MPRAGE image using the interactive software program "ITK-SNAP" (Yushkevich et al., 2006). Anatomical boundaries were determined in coronal sections with the aid of neuroanatomical atlases (Duvernoy, 1997; Mai et al., 2007), and actual segmentation was performed using a previously published protocol (Geuze et al., 2005) in which segmentation was performed from posterior to anterior. The details on hippocampus segmentation were described previously in detail (van Norden et al., 2011b). Volumes were calculated for the left and right hippocampus separately by summing all voxel volumes of the segmented areas. Inter-rater studies on a random sample of $10 \%$ showed an intra-class correlation coefficient for the left hippocampus of 0.73 and for the right hippocampus of 0.79 . Intra-rater studies on this sample showed an intra-class correlation coefficient for the left and right hippocampus of 0.97 and 0.96 , respectively.

We created a mask for the cingulum by applying the white matter atlas (Juelich Histological Atlas white matter labels, provided by FSL) and warped this to the individual T1 volumes (inverse normalization). We counted the number of voxels and multiplied this to voxel volume in order to get the cingulum volume. 
We computed gray (GM) and white-matter (WM) tissue and cerebrospinal fluid (CSF) probability maps using SPM5 unified segmentation routines on the T1 MPRAGE images (SPM5; Wellcome Department of Cognitive Neurology, University College London, UK; Ashburner and Friston, 2005). Total GM, WM, and CSF volumes were calculated by summing all voxel volumes that had a $p>.5$ for belonging to that tissue class. Total brain volume (TBV) was taken as the sum of total GM and WM. Intracranial volume (ICV) was the summation of all tissue classes (total GM, total WM and CSF volume). To normalize for head size, TBV, hippocampal volume and cingulum volume were expressed as a percentage of total ICV.

WML were manually segmented on FLAIR images, and the number of lacunar infarcts was rated according to a standardized protocol (van Norden et al., 2011a). In a random sample of $10 \%$, inter-rater variability for total WML volume yielded an intra-class correlation coefficient of 0.99 , intra- and inter-rater reliability for the lacunar infarcts yielded a weighted kappa of 0.80 and 0.88 .

\section{DTI analysis}

Diffusion data were first preprocessed to detect and correct head and cardiac motion artifacts, using an in-house developed iteratively re-weighted-least-squares algorithm named "PATCH" (www.ru.nl/ neuroimaging/diffusion) (Zwiers, 2010). Corrections of Eddy current and motion artifacts from affine misalignment are performed simultaneously by minimization of the residual diffusion tensor errors (Andersson and Skare, 2002). Next, FA and MD images were calculated using a DTIFit within the Functional MR of the Brain diffusion toolbox, which were then fed into the TBSS pipeline (Smith et al., 2006). The thinning procedure was conducted on the mean FA image to create a common skeleton, which represents the core-structure of the white matter tract. Subsequently this skeleton was thresholded at FA-value 0.3 to include the major white matter tracts and to account for the inter-subject variability. All normalized FA data were then projected onto this skeleton. These skeleton projection factors were then applied to the mean images. These data were then fed into the voxel-wise cross-subject statistics.

In addition, MD and FA values of the cingulum were measured in six bilateral regions of interest (ROIs) in the cingulum bundle as illustrated in Fig. 1. An experienced neurologist (FEdL) blinded to subject information used FSLView (Smith et al., 2004) to overlay an histological atlas (Juelich Histological Atlas; Eickhoff et al., 2005) over the MNI152 standard brain, in order to manually mark out the center coordinates of the ROIs in the different locations of the cingulum and the parahippocampal region (based on neuroanatomy data from the literature) (Schmahmann and Pandya, 2006). We marked out 5 ROIs on a sagittal slice of the cingulum. The cingulum was divided in 4 parts: anterior, middle, posterior and parahippocampal section (Catheline et al., 2010). The center of ROI C was placed at the center of the middle curve of the cingulum fibers, just above the body of the corpus callosum: this is the middle cingulate region. We placed the center of the 2 anterior ROIs (ROI A and B) before the middle curve of the cingulum (around the rostrum and genu of the corpus callosum) and the 2 posterior ROIs (ROI D and E) nearby the dorsal curve of the cingulum fibers (around the splenium of the corpus callosum). The center of the sixth ROI (ROI F) was placed in the parahippocampal cingulum on the medial temporal portion of the cingulum fibers. The center coordinates of each ROI were mirrored with respect to the mid-sagittal plane to obtain these same regions in both cerebral hemispheres. The ROI center coordinates were mapped back to the individual DTI space of each subject using the inverse of the SPM5 unified T1 normalization parameters (Ashburner and Friston, 2005). ROIs were defined as $6 \mathrm{~mm}$ diameter spheres (volume of $0.056 \mathrm{~mL}$ ) around the DTI-space center coordinates. We used a modest ROI size to ensure that all ROIs included only white matter (which was visually checked). FA and MD values within each ROI were averaged. Furthermore, the mean MD was calculated in both hippocampi. All images were visually checked for motion artifacts and co-registration errors, especially for not including perihippocampal CSF.

\section{Other measurements}

The following characteristics were considered possible confounders: age, sex, educational level (Hochstenbach et al., 1998) and depressive symptoms. Depressive symptoms were assessed using the Center of Epidemiologic Studies on Depression Scale (CES-D) (Radloff, 1977).

\section{Structural integrity of the hippocampus}

In order to investigate the role of hippocampal integrity on cingulum integrity, and identify if cingular structural integrity is related to hippocampal pathology, we composed a score for structural hippocampal integrity. As it has been demonstrated that a combination of high diffusivity in the hippocampus and low hippocampal volume is related to conversion to AD in MCI patients, (Kantarci et al., 2005) we compiled a score for hippocampal integrity using both parameters. Both diffusivity and volume were given a score, ranging from 1 (poor: lowest tertile of the volume/highest tertile of MD distribution) to 3 (good; highest tertile of the volume/lowest tertile of the MD distribution) leading to a maximum score between 2 (lowest tertile of the volume and highest tertile of the MD distribution $(1+1)$ : poorest hippocampal integrity) and 6 (highest tertile of the volume and lowest tertile of the MD distribution ( $3+3)$ :best hippocampal integrity). A good hippocampal integrity was defined as a score of $\geq 4$, while a poor integrity was defined as a score of 2 or 3 .

\section{Statistical analysis}

Statistical analyses were performed with SPSS 16.0 for Windows (SPSS Inc., Chicago, IL, USA). Baseline characteristics were summarized as mean \pm standard deviation (SD) or proportions; for skewed variability parameters the median and the inter-quartile range were calculated.

For the TBSS analysis, we assessed voxel-wise correlations between the skeletal DTI parameters (FA and MD) and cognitive performance as mentioned above. Adjustments were made for the same confounders as mentioned above. We applied permutation-based statistical interference tool for non-parametric approach as a part of the Functional MRI of the Brain Software Library, with number of permutation tests set to 5000 (Nichols and Holmes, 2002; Smith et al., 2006). Significant clusters were identified using the threshold-free cluster enhancement with a $p$-value $<0.05$, corrected for multiple comparisons (Smith and Nichols, 2009). In addition, we performed the same analysis with a threshold at $p<0.01$.

For the region-of-interest analyses, we computed regression coefficients of the mean FA and MD of the six ROIs in the cingulum with the four different indices of the RAVLT performance, psychomotor speed, visuospatial memory and MMSE. Adjustments were made for potential confounders including age, sex, educational level, depressive symptoms, normalized total brain volume (NTBV), normalized hippocampal volume (NHV),WML volume and the number of lacunar infarcts. Regression coefficients are presented as standardized $\beta$-values.

To assess whether the structural integrity of the hippocampus modified the relation between cingulum integrity and performance in verbal memory, psychomotor speed, visuospatial memory and MMSE, we performed the previously described linear regression analyses stratified in two groups, good and poor hippocampal integrity. Adjustments were made for the same confounders as mentioned above. To assess whether the groups differed significantly, the correlation coefficients were converted to $Z$-scores using the Fisher r-to- $Z$ 
transformation. The obtained $Z$-scores were used to calculate the $p$-values.

We performed an independent two-sample T-test to assess the differences in MD and FA values of the cingulum when stratified on hippocampal integrity to identify if cingular structural integrity is related to hippocampal pathology. Bonferroni corrections were applied to correct for multiple comparisons.

\section{Results}

The baseline characteristics of the 440 participants are shown in Table 1 . The mean age of the population was 65.2 years (SD 8.9) and $54.3 \%$ were male. The mean MMSE score was 28.2 (SD 1.6). In Table 2 the test scores of the cognitive test battery are shown.

Fig. 2 shows the relation between the voxel-wise analysis of the FA and verbal memory performance ( $p<.05$, corrected for multiple comparisons). For this analysis (and the ROI analysis) we had to exclude 2 participants because of lacking of the data of the CES-D. FA in the cingulum and corpus callosum was positively related to immediate memory, delayed recall, delayed recognition and overall verbal memory performance, especially in the left hemisphere and independent of confounders. By contrast, we found no significant relations with psychomotor speed and neither with visuospatial memory and MMSE (data not shown). We found a similar distribution for the inverse association with MD and verbal memory performance, although these relations were less significant (data are not shown).

Our ROI analysis shows similar results. In Table 3 the relations between the FA of the six ROIs in left and right cingulum and the different cognitive tasks are shown. Significant relations were found between the FA values in the left mid cingulum (ROI C) and immediate memory $(\beta=.09, p=0.041)$ and delayed recognition $(\beta=.11$, $p=0.027)$; in the left ROI D and overall verbal memory performance $(\beta=.10, p=0.027)$, immediate memory $(\beta=.14, p=0.003)$ and delayed recognition $(\beta=.10, p=0.043)$; in the right ROI $D$ and overall verbal memory performance $(\beta=.10, p=0.025)$ and immediate memory $(\beta=.10, p=0.018)$; in the right ROI $\mathrm{E}$ and overall verbal memory performance $(\beta=.09, p=0.037)$ and immediate memory $(\beta=.11, p=0.009)$, independent of confounders. No significant relations were found with psychomotor speed, visuospatial memory and MMSE. Almost no significant results were found between MD values and verbal memory performance (data not shown).

When we stratified the above mentioned relation on a good $(n=291)$ and poor $(n=147)$ hippocampal integrity we did not find

Table 1

Baseline characteristics of the 440 participants.

\begin{tabular}{ll}
\hline Baseline characteristics & \\
Age, years & $65.2(8.9)$ \\
Male $^{*}$ & $239(54.3)$ \\
Participants with only primary education $^{*}$ & $41(9.3)$ \\
MMSE & $28.2(1.6)$ \\
CES-D & $11.1(9.4)$ \\
Participants with depressive symptoms ${ }^{*}, \mathrm{a}$ & $151(34.3)$ \\
& \\
Neuroimaging characteristics & \\
TBV (mL) & $1098.6(119.0)$ \\
ICV (mL) & $1674.7(157.4)$ \\
Normalized hippocampal volume & \\
Normalized cingular volume & \\
White matter volume (mL) & $0.41(0.06)$ \\
WML volume (mL) & \\
Presence of lacunar infarcts & \\
\end{tabular}

MMSE, minimal mental state examination; CES-D, Centre of Epidemiological Studies on Depression Scale; TBV, total brain volume; ICV, intracranial volume; WML, white matter lesions.

Data represent $n$ of subjects $(\%)^{*}$, mean (SD), median ${ }^{\dagger}$ (inter-quartile range) or percentage regarding ICV ${ }^{\ddagger}(\mathrm{SD})$.

a Defined as CES-D scores $\geq 16$ and/or the current use of anti-depressive medication.
Table 2

Cognitive test scores.

\begin{tabular}{ll}
\hline RAVLT: number of words recalled & \\
Immediate recall trial $1(n=439)$ & $5.2(1.8)$ \\
Immediate recall trial $2(n=439)$ & $7.4(2.3)$ \\
Immediate recall trial $3(n=439)$ & $8.7(2.6)$ \\
Total of immediate recall trial $1-3(n=439)$ & $21.3(6.1)$ \\
Delayed recall $(n=439)$ & $6.0(3.1)$ \\
Delayed recognition $(n=436)$ & $27.1(3.2)$ \\
Stroop test (time in seconds) & \\
Trial 1 (words) $(n=427)$ & $25.5(6.1)$ \\
Trial 2 (colors) $(n=427)$ & $32.9(7.5)$ \\
Trial 3 (concept-shifting) $(n=427)$ & $62.8(20.9)$ \\
Paper and pencil memory scanning task (time in seconds) & \\
1 character $(n=428)$ & $44.3(13.5)$ \\
2 characters $(n=428)$ & $61.2(18.7)$ \\
3 characters $(n=428)$ & $76.0(26.2)$ \\
Symbol digit substitution task (no. in $60 \mathrm{~s})(n=437)$ & $27.7(9.7)$ \\
Rey complex figure test (range 0-36) & \\
Copy trial $(n=435)$ & $33.5(3.4)$ \\
Immediate recall trial $(n=430)$ & $18.1(6.8)$ \\
Delayed recall trial $(n=416)$ & $18.1(6.7)$
\end{tabular}

RAVLT, Rey Auditory Verbal Learning Test.

Numbers represent means (SD).

significant differences in the correlations coefficients between both groups, except for the relation with immediate memory and FA in the left ROI B (results are not shown).

Table 4 shows cingulum integrity (expressed as mean MD and FA values of the six ROIs in the left and right cingulum) stratified on hippocampal integrity. The MD and FA values of all ROIs differed significantly between both groups (good and poor hippocampal integrity), except for the FA values in ROI E and F. Our results showed that the cingular integrity is significantly lower in participants with a poor hippocampal integrity than in participants with a good hippocampal integrity.

\section{Discussion}

In this study we investigated the relation between cingulum integrity and verbal memory performance by as well an ROI based approach as a TBSS analysis of the cingulum in 503 non-demented elderly with SVD. We demonstrated that the microstructural integrity of the cingulum is specifically related to verbal memory performance, independent of confounders, including hippocampal volume and coexisting SVD. Furthermore we found that when the integrity of the hippocampus is disrupted, the cingulum integrity is impaired as well.

To the best of our knowledge this is the first study investigating the relation between the microstructural integrity of the cingulum and verbal memory function in a large group of non-demented, independently living elderly with cerebral SVD. Our data show that the microstructural integrity of the cingulum beyond the detection limit of conventional MRI is related to episodic memory formation. On conventional MRI only less than $6 \%$ of the ROIs in the cingulum of our study population, showed the presence of visible WML, strengthening our assumption that we indeed investigated the earliest structural changes in the cingulum bundle integrity.

Our finding is in line with the results of previous studies in patients with $\mathrm{MCI}$ or $\mathrm{AD}$ showing that loss of structural integrity of the cingulum is associated to impaired verbal memory performance (Bozzali et al., 2012; Fellgiebel et al., 2005; Kantarci et al., 2011; Mielke et al., 2009; Sexton et al., 2010).

Furthermore we found that participants with a poor hippocampal integrity (low volume and high MD) had a significantly poorer structural integrity of the cingulum (high MD as well as low FA) than participants with a good hippocampal integrity (high volume and low MD). In addition we found that the relation between cingulum integrity and verbal memory performance did not differ 
Table 3

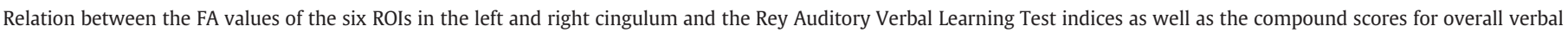
memory performance, psychomotor speed, visuospatial memory and MMSE.

\begin{tabular}{|c|c|c|c|c|c|c|c|c|c|c|c|c|}
\hline & \multirow{2}{*}{\multicolumn{2}{|c|}{ FA ROI A }} & \multirow{2}{*}{\multicolumn{2}{|c|}{$\frac{\text { To anterior } \leftarrow}{\text { FA ROI B }}$}} & \multirow{2}{*}{\multicolumn{2}{|c|}{$\begin{array}{l}\text { Mid cingulum } \\
\text { FA ROI C } \\
\end{array}$}} & \multirow{2}{*}{\multicolumn{2}{|c|}{$\begin{array}{l}\rightarrow \text { To posterior } \\
\text { FA ROI D }\end{array}$}} & \multirow{2}{*}{\multicolumn{2}{|c|}{ FA ROI E }} & \multirow{2}{*}{\multicolumn{2}{|c|}{$\frac{\text { Parahippocampal }}{\text { FA ROI F }}$}} \\
\hline & & & & & & & & & & & & \\
\hline & Left & Right & Left & Right & Left & Right & Left & Right & Left & Right & Left & Right \\
\hline Overall verbal memory performance ${ }^{a}$ & .03 & -.01 & .04 & -.01 & .08 & -.01 & $.10^{* *}$ & $.10^{* *}$ & .06 & $.09^{* *}$ & -.01 & -.02 \\
\hline Immediate memory & .06 & .01 & .05 & -.02 & $.09^{* *}$ & -.01 & $.14^{*}+\ddagger$ & $.10^{* *}$ & .07 & $.11^{*}$ & -.01 & -.04 \\
\hline Delayed recall & .01 & -.01 & .03 & .01 & .05 & .01 & .06 & .08 & .05 & .06 & -.01 & .01 \\
\hline Delayed recognition & .06 & .05 & .06 & .04 & $.11^{* *}$ & .07 & $.10^{* *}$ & .09 & .06 & .03 & .05 & -.04 \\
\hline Psychomotor speed $^{\mathrm{a}}$ & -.09 & -.09 & -.06 & -.06 & .01 & -.08 & .01 & -.02 & -.04 & .05 & -.02 & -.07 \\
\hline Visuospatial memory $^{\mathrm{a}}$ & -.01 & -.04 & .07 & -.07 & .03 & -.03 & .08 & .06 & .02 & .04 & .01 & -.05 \\
\hline MMSE & -.06 & -.06 & -.01 & -.05 & .03 & .02 & .01 & .01 & .02 & .03 & -.05 & .01 \\
\hline
\end{tabular}

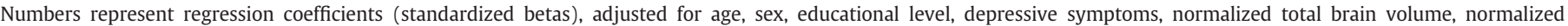
hippocampal volume, WML volume and the number of lacunar infarcts.

After Bonferroni correction: ${ }^{\dagger} p<0.01 ;{ }^{\ddagger} p<0.05$.

$* p<0.01$

** $p<0.05$.

a Compound scores.

significantly between both groups (good and poor hippocampal integrity). These results might suggest that hippocampal integrity is not an intermediate in this relation. There is still much debate whether, in general, WM pathology is related to, or independent of, GM pathology. There are three main hypotheses, one suggesting that microstructural WM changes occur as a result of Wallerian degeneration (Coleman, 2005), meaning that WM pathology is preceded by GM pathology. In contrast, the retrogenesis theory proposes that loss of WM integrity is the result of myelin breakdown that occurs in the reverse order to myelogenesis (Bartzokis, 2004; Reisberg et al., 2002). Furthermore there is evidence that small vessel disease can cause WM damage occurring as a result of oligodendrocyte death and reactive gliosis. (Englund and Brun, 1990). Our results are not able to distinguish between these theories, also because of the cross-sectional design of our study.

With our TBSS analysis we found that the microstructural integrity of the mid-anterior part of the cingulum is related to impaired verbal memory, while our less precise ROI analysis found the mid-posterior part to be involved. As mentioned before, most studies in MCI and AD mainly found involvement of the posterior cingulum. However most of them included only a few ROIs in the cingulum, did not perform a TBSS analysis on the cingulum and investigate the cingulum integrity in patients with already $\mathrm{MCI}$ and/or AD. Probably our results suggest that loss of structural integrity starts in the middle-anterior parts of the cingulum and maybe further spreading to the posterior parts, near medial temporal lobe structures, with progression of verbal memory impairment and ultimately dementia. Future studies are needed to describe the possible spread over time of the pathological process throughout the cingulum. A follow-up of the RUN DMC study is already being executed, to further investigate this.

Our findings show that cingulum integrity is specifically related to verbal episodic memory. We did not find relations between cingulum integrity and psychomotor speed, visuospatial memory and MMSE. Although a recent study did find associations between (posterior) cingulum integrity and attention/executive functioning and visuospatial memory (Kantarci et al., 2011), this discrepancy may be due to the limited additional adjustments performed in the latter study. Furthermore their study population differed from ours. Of their 220 participants, 149 had MCI, while our study population had a mean MMSE of 28.1 and only a small percentage (10\%) sufficed the diagnosis $\mathrm{MCI}$ according to the definition of van der Elst (van der Elst et al., 2005). As a result it is likely that more damage to the structural

Table 4

Mean MD and FA of the six ROIs in the left and right cingulum stratified on hippocampal integrity.

\begin{tabular}{|c|c|c|c|c|c|c|}
\hline & \multicolumn{6}{|c|}{ Hippocampal integrity } \\
\hline & \multirow{2}{*}{$\frac{\text { Good }}{\mathrm{MD}}$} & \multicolumn{2}{|l|}{ Poor } & \multirow{2}{*}{$\frac{\text { Good }}{\text { FA }}$} & \multicolumn{2}{|l|}{ Poor } \\
\hline & & MD & $p$-Value & & $\mathrm{FA}$ & $p$-Value \\
\hline \multicolumn{7}{|c|}{ ROI A: anterior } \\
\hline Left & $8.29(0.74)$ & $8.83(1.90)$ & $<0.01$ & $0.41(0.10)$ & $0.38(0.10)$ & $<0.05$ \\
\hline Right & $8.22(0.60)$ & $8.75(1.91)$ & $<0.01$ & $0.39(0.08)$ & $0.37(0.08)$ & $<0.01$ \\
\hline \multicolumn{7}{|c|}{ ROI B: anterior } \\
\hline Left & $8.24(0.93)$ & $8.82(1.69)$ & $<0.001$ & $0.42(0.10)$ & $0.37(0.10)$ & $<0.001$ \\
\hline Right & $8.12(0.67)$ & $8.85(1.82)$ & $<0.001$ & $0.42(0.09)$ & $0.39(0.09)$ & $<0.001$ \\
\hline \multicolumn{7}{|c|}{ ROI C: mid cingulum } \\
\hline Left & $7.68(0.55)$ & $8.32(1.76)$ & $<0.001$ & $0.53(0.09)$ & $0.47(0.10)$ & $<0.001$ \\
\hline Right & $7.78(0.55)$ & $8.40(1.54)$ & $<0.001$ & $0.52(0.09)$ & $0.47(0.09)$ & $<0.001$ \\
\hline \multicolumn{7}{|c|}{ ROI D: superior-posterior } \\
\hline Left & $7.80(0.42)$ & $8.28(1.84)$ & $<0.01$ & $0.54(0.07)$ & $0.51(0.09)$ & $<0.001$ \\
\hline Right & $7.76(0.48)$ & $8.21(1.63)$ & $<0.01$ & $0.51(0.07)$ & $0.49(0.07)$ & $<0.01$ \\
\hline \multicolumn{7}{|c|}{ ROI E: inferior-posterior } \\
\hline Left & $7.82(0.47)$ & $8.10(1.80)$ & $<0.05$ & $0.45(0.07)$ & $0.46(0.07)$ & NS \\
\hline Right & $7.98(0.55)$ & $8.41(1.71)$ & $<0.001$ & $0.39(0.07)$ & $0.38(0.07)$ & NS \\
\hline \multicolumn{7}{|c|}{ ROI F: parahippocampal } \\
\hline Left & $8.57(0.74)$ & $9.01(0.81)$ & $<0.001$ & $0.25(0.06)$ & $0.25(0.07)$ & NS \\
\hline Right & $8.74(0.71)$ & $9.27(1.00)$ & $<0.001$ & $0.25(0.08)$ & $0.25(0.07)$ & NS \\
\hline
\end{tabular}

MD, mean diffusivity, expressed as $10^{-4} \mathrm{~mm}^{2} / \mathrm{s}$. FA, fractional anisotropy. NS, not significant.

Numbers represent means (SD). 
integrity of the cingulum and MTL structures like the hippocampus has occurred in the study population of Kantarci.

The reason for not finding a relation between DTI parameters and visuospatial memory in our study may be due to the fact that memory performance based on the Rey Complex Figure Test (RCFT) may in part rely on motor learning. Therefore the RCFT cannot be regarded as a pure measure of spatial memory, as part of episodic memory, which as a result may be less dependent of the hippocampal memory circuit.

In our study also the corpus callosum is involved in verbal memory performance, as shown by our TBSS analysis. DTI abnormalities in $\mathrm{MCI}$ and $\mathrm{AD}$ have been found in the corpus callosum (Head et al., 2004; Shim et al., 2008). A study of Christman and Propper (2001) showed that episodic memory is at least in part dependent on interhemispheric interaction. Furthermore it has been reported that older adults show reduced hemispheric asymmetry in episodic memory functioning (Madden et al., 1999). Disruption of the corpus callosum can lead to impaired interhemispheric interaction, resulting in impaired (verbal) memory performance.

With respect to lateralization effects, our study showed the strongest relation to verbal memory in the left hemisphere. As would be expected from a verbal task, the dominant (left) hemisphere, containing the language centers (Broca and Wernicke), is more strongly involved in performing the RALVT, as most of our participants were right handed.

Still, a few methodological considerations need to be addressed. Although our data are derived from the largest DTI study on the cingulum thus far, our study design is cross-sectional, which prevents us from drawing conclusions with respect to causality. Data of our follow-up study (van Norden et al., 2011a), which is currently underway, might help to further investigate the structural integrity of the cingulum in relation with structural changes in the medial temporal lobe structures and in the development of cognitive decline and dementia. Furthermore, we used an atlas to identify the cingulum bundle, and manually placed the ROIs in order to avoid GM and WM of other tracts to blur our DTI measures. Although the placement of the atlas was visually checked and the placement of the ROIs was performed by an experienced neurologist, blinded to clinical data, this method can be somewhat subjective and can make interpretation between studies difficult.

A major strength of our study is the fact that it is a large, single-center study with a response rate of over $70 \%$. All MRI data were acquired on a single scanner in a similar way, and the hippocampus and WML were assessed volumetrically in a reliable, sensitive and objective way by two trained experts, who were blinded to all clinical data. Furthermore, extensive adjustments were made for possible confounders. Moreover, cognitive function was assessed by only two investigators using a standardized cognitive battery.

In conclusion, this study demonstrates that microstructural integrity of the cingulum, assessed by TBSS analysis and a ROI-based approach, is specifically related to episodic memory function, notably verbal memory, in non-demented elderly with SVD. Furthermore, we showed that cingulum integrity is significantly lower in participants with a poor hippocampal integrity than in participants with a good hippocampal integrity.

Consequently, our findings show that DTI, by using a ROI-based and TBSS approach, is a sensitive diagnostic tool to detect early microstructural changes in the cingulum, which are related to impaired verbal memory, before structural changes of cingulum can be detected on conventional MRI. Future studies should prospectively investigate the predictive value of DTI parameters of the cingulum in relation to cognitive consequences of SVD and incident dementia. If the predictive value is proven, DTI of the cingulum could possibly be a surrogate marker for development of cognitive decline and dementia and could be a starting point for therapeutic trials aiming to prevent disease progression.

\section{Disclosure statement}

No conflicts of interest are present.

\section{Acknowledgments}

F.E.d.L was supported by Dutch Brain Foundation (H04-12), clinical fellowship Netherlands Organization Scientific Research (40-0070397-07197), and “Internationale Stichting Alzheimer Onderzoek."

R.P.C.K. was supported by a VIDI innovational grant from the Netherlands Organization for Scientific Research (NWO, no. 452-08-005).

\section{References}

Acosta-Cabronero, J., Williams, G.B., Pengas, G., Nestor, P.J., 2010. Absolute diffusivities define the landscape of white matter degeneration in Alzheimer's disease. Brain 133, 529-539.

American Psychiatric Association, 2000. Diagnostic and Statistical Manual of Mental Disorders, 4th ed. text revision. Washington DC.

Andersson, J.L., Skare, S., 2002. A model-based method for retrospective correction of geometric distortions in diffusion-weighted EPI. Neuroimage 16, 177-199.

Ashburner, J., Friston, K.J., 2005. Unified segmentation. Neuroimage 26, 839-851.

Bartzokis, G., 2004. Age-related myelin breakdown: a developmental model of cognitive decline and Alzheimer's disease. Neurobiol. Aging 25, 5-18.

Basser, P.J., Mattiello, J., LeBihan, D., 1994. Estimation of the effective self-diffusion tensor from the NMR spin echo. J. Magn. Reson. B 103, 247-254.

Bozzali, M., Guilietti, G., Basile, B., Serra, L., Spanò, B., Perri, R., Giubilei, F., Marra, C., Caltagirone, C., Cercignani, M., 2012. Damage to the cingulum contributes to Alzheimer's disease pathophysiology by deafferentation mechanism. Hum. Brain Mapp 33, 1295-1308.

Brun, A., Englund, E., 1986. A white matter disorder in dementia of the Alzheimer type: a pathoanatomical study. Ann. Neurol. 19, 253-262.

Catheline, G., Periot, O., Amirault, M., Braun, M., Dartigues, J.F., Auriacombe, S., Allard, M., 2010. Distinctive alterations of the cingulum bundle during aging and Alzheimer's disease. Neurobiol. Aging 31, 1582-1592.

Charlton, R.A., Barrick, T.R., McIntyre, D.J., Shen, Y., O'Sullivan, M., Howe, F.A., Clark, C.A., Morris, R.G., Markus, H.S., 2006. White matter damage on diffusion tensor imaging correlates with age-related cognitive decline. Neurology 66, 217-222.

Choo, I.H., Lee, D.Y., Oh, J.S., Lee, J.S., Lee, D.S., Song, I.C., Youn, J.C., Kim, S.G., Kim, K.W. Jhoo, J.H., Woo, J.I., 2010. Posterior cingulate cortex atrophy and regional cingulum disruption in mild cognitive impairment and Alzheimer's disease. Neurobiol. Aging 31, 772-779.

Christman, S.D., Propper, R.E., 2001. Superior episodic memory is associated with interhemispheric processing. Neuropsychology 15, 607-616.

Chua, T.C., Wen, W., Chen, X., Kochan, N., Slavin, M.J., Troller, J.N., Brodaty, H., Sachdev P.S., 2009. Diffusion tensor imaging of the posterior cingulate is a useful biomarker of mild cognitive impairment. Am. J. Geriatr. Psychiatry 17, 602-613.

Coleman, M., 2005. Axon degeneration mechanisms: commonality amid diversity. Nat. Rev. Neurosci. 6, 889-898.

de Groot, J.C., de Leeuw, F.E., Oudkerk, M., van Gijn, J., Hofman, A., Jolles, J., Breteler, M.M., 2000. Cerebral white matter lesions and cognitive function: The Rotterdam Scan Study. Ann. Neurol. 47, 145-151.

de Groot, J.C., de Leeuw, F.E., Oudkerk, M., van Gijn, J., Hofman, A., Jolles, J., Breteler, M.M., 2002. Periventricular cerebral white matter lesions predict rate of cognitive decline. Ann. Neurol. 52, 335-341.

de Leeuw, F.E., de Groot, J.C., Achten, E., Oudkerk, M., Ramos, L.M., Heijboer, R., Hofman, A., Jolles, J., van Gijn, J., Breteler, M.M., 2001. Prevalence of cerebral white matter lesions in elderly people: a population based magnetic resonance imaging study. The Rotterdam Scan Study. J. Neurol. Neurosurg. Psychiatry 70, 9-14.

Duvernoy, H., 1997. The Human Hippocampus: Functional Anatomy, Vascularization and Serial Sections with MRI (Hardcover). Springer-Verlag, New York.

Eickhoff, S.B., Stephan, K.E., Mohlberg, H., Grefkes, C., Fink, G.R., Amunts, K., Zilles, K., 2005. A new SPM toolbox for combining probabilistic cytoarchitectonic maps and functional imaging data. Neuroimage 25, 1325-1335.

Englund, E., Brun, A., 1990. White matter changes in dementia of Alzheimer's type: the difference in vulnerability between cell compartments. Histopathology 16, 433-439.

Erkinjuntti, T., 2002. Subcortical vascular dementia. Cerebrovasc. Dis. 13 (Suppl. 2), 58-60.

Fellgiebel, A., Muller, M.J., Wille, P., Dellani, P.R., Scheurich, A., Schmidt, L.G., Stoeter, P. 2005. Color-coded diffusion-tensor-imaging of posterior cingulate fiber tracts in mild cognitive impairment. Neurobiol. Aging 26, 1193-1198.

Folstein, M.F., Folstein, S.E., McHugh, P.R., 1975. "Mini-mental state". A practical method for grading the cognitive state of patients for the clinician. J. Psychiatr. Res. 12, 189-198.

Geuze, E., Vermetten, E., Bremner, J.D., 2005. MR-based in vivo hippocampal volumetrics: II. Findings in neuropsychiatric disorders. Mol. Psychiatry 10, 160-184.

Gouw, A.A., Seewann, A., Vrenken, H., van der Flier, W.M., Rozemuller, J.M., Barkhof, F, Scheltens, P., Geurts, J.J., 2008. Heterogeneity of white matter hyperintensities in Alzheimer's disease: post-mortem quantitative MRI and neuropathology. Brain 131, 3286-3298.

Head, D., Buckner, R.L., Shimony, J.S., Williams, L.E., Akbudak, E., Conturo, T.E., McAvoy M., Morris, J.C., Snyder, A.Z., 2004. Differential vulnerability of anterior white matter in nondemented aging with minimal acceleration in dementia of the Alzheimer type: evidence from diffusion tensor imaging. Cereb. Cortex 14, 410-423. 
Hochstenbach, J., Mulder, T., van Limbeek, J., Donders, R., Schoonderwaldt, H., 1998. Cognitive decline following stroke: a comprehensive study of cognitive decline following stroke. J. Clin. Exp. Neuropsychol. 20, 503-517.

Houx, P.J., Jolles, J., Vreeling, F.W., 1993. Stroop interference: aging effects assessed with the Stroop Color-Word Test. Exp. Aging Res. 19, 209-224.

Kantarci, K., Petersen, R.C., Boeve, B.F., Knopman, D.S., Weigand, S.D., O'Brien, P.C., Shiung, M.M., Smith, G.E., Ivnik, R.J., Tangalos, E.G., Jack Jr., C.R., 2005. DWI predicts future progression to Alzheimer disease in amnestic mild cognitive impairment. Neurology 64, 902-904.

Kantarci, K., Senjem, M.L., Avula, R., Zhang, B., Samikoglu, A.R., Weigand, S.D. Przybelski, S.A., Edmonson, H.A., Vemuri, P., Knopman, D.S., Boeve, B.F., Ivnik, R.J. Smith, G.E., Petersen, R.C., Jack, C.R., 2011. Diffusion tensor imaging and cognitive function in older adults with no dementia. Neurology 77, 26-34.

Kiuchi, K., Morikawa, M., Taoka, T., Nagashima, T., Yamauchi, T., Makinodan, M., Norimoto, K., Hashimoto, K., Kosaka, J., Inoue, M., Kichikawa, K., Kishimoto, T., 2009. Abnormalities of the uncinate fasciculus and posterior cingulated fasciculus in mild cognitive impairment and early Alzheimer's disease: a diffusion tensor tractography study. Brain Res. 1287, 184-191.

Lezak, M.D., 1976. Neuropsychological Assessment, 3rd ed. Oxford University Press, New York.

Madden, D.J., Turkington, T.G., Provenzale, J.M., Denny, L.L., Hawk, T.C., Gottlob, L.R., Coleman, R.E., 1999. Adult age differences in the functional neuroanatomy of verbal recognition memory. Hum. Brain Mapp. 7, 115-135.

Mai, J., Paxinos, G., Voss, T., 2007. Atlas of the Human Brain, 3rd ed. Elsevier, San Diego, CA.

Mielke, M.M., Kozauer, N.A., Chan, K.C., George, M., Toroney, J., Zerrate, M., BandeenRoche, K., Wang, M.C., Vanzijl, P., Pekar, J.J., Mori, S., Lyketsos, C.G., Albert, M., 2009. Regionally-specific diffusion tensor imaging in mild cognitive impairment and Alzheimer's disease. Neuroimage 15, 47-55.

Müller, M., Greverus, D., Weibrich, C., Dellani, P.R., Scheurich, A., Stoeter, P., Fellgiebel, A., 2007. Diagnostic utility of hippocampal size and mean diffusivity in amnestic MCI. Neurobiol. Aging 28, 398-403.

Nakata, Y., Sato, N., Nemoto, K., Abe, O., Shikakura, S., Arima, K., Furuta, N., Uno, M., Hirai, S., Masutani, Y., Ohtomo, K., Barkovich, A.J., Aoki, S., 2008. Diffusion abnormality in the posterior cingulum and hippocampal volume: correlation with disease progression in Alzheimer's disease. Magn. Reson. Imaging 27, 347-354.

Nichols, T.E., Holmes, A.P., 2002. Nonparametric permutation tests for functiona neuroimaging: a primer with examples. Hum. Brain Mapp. 15, 1-25.

Osterrieth, P.A., 1944. Le test de copie dune figure complexe. Contribution a l'étude de la perception et de la mémoire. Arch. Psychol. 30, 206-353.

Pierpaoli, C., Jezzard, P., Basser, P.J., Barnett, A., Di Chiro, G., 1996. Diffusion tensor MR imaging of the human brain. Radiology 201, 637-648.

Radloff, L.S., 1977. The CES-D Scale: a self-report depression scale for research in the general population. Appl. Psychol. Meas. 1, 385-401.

Reisberg, B., Franssen, E.H., Souren, L.E., Auer, S.R., Akram, I., Kenowsky, S., 2002. Evidence and mechanisms of retrogenesis in Alzheimer's and other dementias: management and treatment import. Am. J. Alzheimers Dis. Other Demen. 17, 202-212.

Schmahmann, J.D., Pandya, D.N., 2006. Fiber Pathways of the Brain. Oxford University Press, New York.

Sepulcre, J., Masdeu, J.C., Sastre-Garriga, J., Goni, J., Velez-de-Mendizabal, N., Duque, B. Pastor, M.A., Bejarano, B., Villoslada, P., 2008. Mapping the brain pathways of declarative verbal memory: Evidence from white matter lesions in the living human brain. Neuroimage 42, 1237-1243.

Sexton, C.E., Mackay, C.E., Lonie, J.A., Bastin, M.E., Terrière, E., O'Carroll, R.E., Ebmeier K.P., 2010. MRI correlates of episodic memory in Alzheimer's disease, mild cognitive impairment, and healthy aging. Psychiatry Res. 184, 57-62.
Shim, Y.S., Yoon, B., Shon, Y.M., Ahn, K.J., Yang, D.W., 2008. Difference of the hippocampal and white matter microalterations in MCI patients according to the severity of subcortical vascular changes: neuropsychological correlates of diffusion tensor imaging. Clin. Neurol. Neurosurg. 110, 552-561.

Smith, S.M., Nichols, T.E., 2009. Threshold-free cluster enhancement: addressing problems of smooting, threshold dependence and localisation in cluster inference. Neuroimage 44, 83-98.

Smith, S.M. Jenkinson, M., Woolrich, M.W Beckmann, C.F., Behrens, T.E., JohansenBerg, H., Bannister, P.R., De Luca, M., Drobnjak, I., Flitney, D.E., Niazy, R.K., Saunders, J., Vickers, J., Zhang, Y., De Stefano, N., Brady, J.M., Matthews, P.M., 2004. Advances in functional and structural MR image analysis and implementation as FSL. Neuroimage 23, S208-S219.

Smith, S.M., Jenkinson, M., Johansen-Berg, H., Rueckert, D., Nichols, T.E., Mackay, C.E., Watkins, K.E., Ciccarelli, O., Cader, M.Z., Matthews, P.M., Behrens, T.E., 2006. Tract-based spatial statistics: voxelwise analysis of multi-subject diffusion data. Neuroimage 31, 1487-1505.

Sternberg, S., 1969. Memory-scanning: mental processes revealed by reaction-time experiments. Am. Sci. 57, 421-457.

Stroop, J.R., 1935. Studies of interference in serial verbal reactions. J. Exp. Psychol. 18, 643-662.

Vakil, E., Blachstein, H., 1993. Rey auditory-verbal learning test: structure analysis. J. Clin. Psychol. 49, 883-890.

van der Elst, W., van Boxtel, M.P., van Breukelen, G.J., Jolles, J., 2005. Rey's verbal learning test: normative data for 1855 healthy participants aged $24-81$ years and the influence of age, sex, education, and mode of presentation. J. Int. Neuropsychol. Soc. 11, 290-302.

van Norden, A.G.W., de Laat, K.F., Gons, R.A., van Uden, I.W., van Dijk, E.J., van Oudheusden, L.J., Esselink, R.A., Bloem, B.R., van Engelen, B.G., Zwarts, M.J., Tendolkar, I., Olde-Rikkert, M.G., van der Vlugt, M.J., Zwiers, M.P., Norris, D.G., de Leeuw, F.E., 2011a. Causes and consequences of cerebral small vessel disease. The RUN DMC study: a prospective cohort study. Study rationale and protocol. BMC Neurol. http://dx.doi.org/10.1186/1471-2377-11-29(Epub).

van Norden, A.G.W., de Laat, K.F., Fick, I., van Uden, I.W.M., van Oudheusden, L.J.B. Gons, R.A.R., Norris, D.G., Zwiers, M.P., Kessels, R.P.C., de Leeuw, F.E., 2011b. Diffusion tensor imaging of the hippocampus and verbal memory performance: the RUN DMC Study. Hum. Brain Mapp. http://dx.doi.org/10.1002/hbm.21231(Epub).

van Norden, A.G., de Laat, K.F., van Dijk, E.J., van Uden, I.W., van Oudheusden, L.J., Gons, R.A., Norris, D.G., Zwiers, M.P., de Leeuw, F.E., 2011c. Diffusion tensor imaging and cognition in cerebral small vessel disease The RUN DMC study. Biochim. Biophys. Acta. http://dx.doi.org/10.1016/j.bbadis.2011.04.008(Epub).

Vermeer, S.E., Prins, N.D., den Heijer, T., Hofman, A., Koudstaal, P.J., Breteler, M.M., 2003. Silent brain infarcts and the risk of dementia and cognitive decline. N. Engl. J. Med. 348, 1215-1222.

Xie, S., Xiao, J.X., Wang, Y.H., Wu, H.K., Gong, G.L., Jiang, X.X., 2005. Evaluation of bilateral cingulum with tractography in patients with Alzheimer's disease. Neuroreport $16,1275-1278$

Yushkevich, P.A., Piven, J., Hazlett, H.C., Smith, R.G., Ho, S., Gee, J.C., Gerig, G., 2006. User-guided 3D active contour segmentation of anatomical structures: significantly improved efficiency and reliability. Neuroimage 31, 1116-1128.

Zhang, Y., Schuff, N., Jahng, G.H., Bayne, W., Mori, S., Schad, L., Mueller, S., Du, A.T., Kramer, J.H., Yaffe, K., Chui, H., Jagust, W.J., Miller, B.L., Weiner, M.W., 2007. Diffusion tensor imaging of cingulum fibers in mild cognitive impairment and Alzheimer disease. Neurology 68, 13-19.

Zwiers, M.P., 2010. Patching cardiac and head motion artefacts in diffusion-weighted images. Neuroimage 53, 565-575. 